\title{
Model-Driven Resilience Assessment of Modifications to HPC Infrastructures
}

\author{
Christian Straube and Dieter Kranzlmüller \\ Munich Network Management (MNM) Team, \\ Ludwig-Maximilians-Universität München, Germany, \\ Leibniz Supercomputing Center (LRZ), Munich, Germany \\ straube@nm.ifi.lmu.de
}

\begin{abstract}
The increasing influence of surrounding factors on HPC infrastructure operations, e.g., increasing cost pressure or evolving power consumption, requires an analysis of the indented effects of modifications in the context of the entire HPC infrastructure and its capabilities, before this modification is further (costly) explored or executed actually. Therefore, a meta model is provided that enables a domain-spanning capability effect analysis, which respects the complex component interplay of contemporary HPC infrastructures. The meta model in turn is used as basis for the model-driven development of tooling support.
\end{abstract}

Keywords: Infrastructure, Availability, Reliability, Modeling, Optimization.

\section{Introduction}

Conventional cause-effect-analysis (CEA) is a distinguished tool to analyze the (theoretical) technical effects of a modification in a well-defined specific area, e.g., the impact of using symmetric active/active redundancy (cause) on reliability (effect). The findings are used to alter the modification or to support the decision-making about its execution. Nevertheless, development in the surroundings and in the layout of contemporary HPC infrastructures require an extension of modification planning and evaluation: before it is investigated how a particular effect in a specific area can be achieved, it must be decided whether this intended effect and its consequences are reasonable at all in the context of the entire HPC infrastructure. Especially from an operations point of view, this decision is made upon the expected effects to the HPC infrastructure's capabilities, e.g., the "capability to consume less energy", and consequently, we call the analyzed effects capability effects. The resulting capability effect analysis is based upon two pillars: a complex multi-domain optimization problem and the HPC infrastructure components interaction.

The optimization problem is defined by the evolution in several surrounding factors of HPC infrastructures, like increasing cost pressure (cost), evolving power consumption (energy) or ever more demanding scientific calculations

D. an Mey et al. (Eds.): Euro-Par 2013 Workshops, LNCS 8374, pp. 707-716, 2014.

(C) Springer-Verlag Berlin Heidelberg 2014 
(performance). For instance, increasing redundancy to address short-time breakdown (reliability) [1, simultaneously increases energy consumption and degrades performance due to redundancy overhead, leading to an optimization problem between the dimensions reliability, energy, and performance 2. Consequently, capability effect analysis must be domain-spanning, to weigh out tradeoffs in the context of the entire HPC infrastructure as basis for the decisionmaking about the modification's investigation and execution.

Considering component interactions is important since HPC infrastructures provide their functionality by a qualitative and quantitative complex component interplay, which hardens separating the specific contribution of a particular component. Additionally, most characteristics are combined and influenced by different aspects, even within a single domain, e.g., system performance includes computing cores as well as communication, interconnect, and I/O performance 34. Hence, effects induced by local modifications on a single component can quickly cascade and affect the HPC infrastructure partly or completely in an unpredictable way. Consequently, capability effect analysis must cover effect cascading within the entire HPC infrastructure, and not only a particular component or a (small) subset.

Capability effect analysis applies the generic CEA concept with different input causes, as depicted in Figure 1 on the left, conventional CEA investigates the correlation of a planned modification (cause: $P M_{i}$ ) and its (theoretical) technical effect within a single dimension (effect: $T E_{i}$ ). In contrast, the capability effect analysis on the right, uses the intended effects $\left(I E_{j}\right)$ of the planned modifications from all considered dimensions and the component interaction as causes to calculate a capability effect $\left(C E_{z}\right)$ vector that is used to weigh out trade-offs and to decide, whether a modification is further explored or executed actually (dashed arrow).

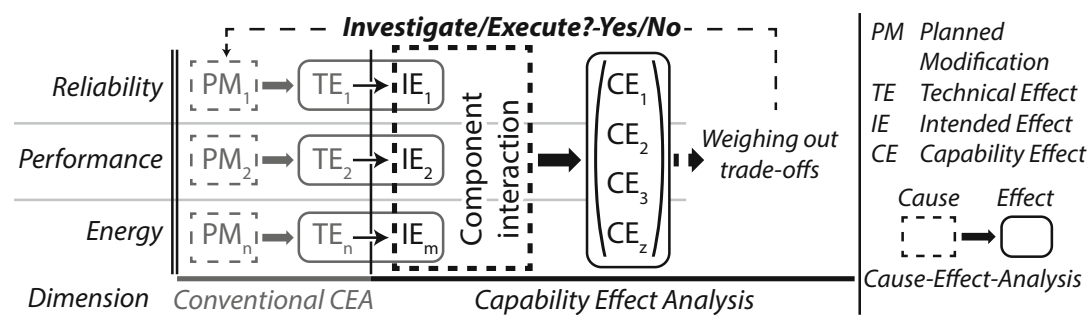

Fig. 1. Difference of conventional cause-effect-analysis and capability effect analysis

In summary, decision-making about a planned modification's execution should be built upon systematic planning and analysis of its expected or intended capability effects [56]: the modification is only executed if its intended capability effects will lead to an improvement for the aforementioned optimization problem. How the modification is executed is neglected in this consideration and passed over, e.g., to conventional CEA approaches. Applying this to the example above, redundancy should be increased (modified) only if it actually improves 
reliability (effect) and the increasing energy consumption and performance losses do not outweigh the benefits (effect optimization problem). How redundancy is concretely implemented is examined by one of the plenty existing approaches.

Although there are a lot of distinguished and mature approaches to conduct conventional CEA for resilience aspects, none of them can be used for capability effect analysis as explained in Section 2, In [7/8, a meta model is presented, which is dedicated to provide capability effect analysis functionality in the above described manner, but lacking resilience aspects. The objective of this paper is to extend the meta model by resilience aspects in three steps: 1) Section 3.1 provides a condensed overview of relevant quantification and modeling methodologies, 2) Section 3.2 extracts their commonalities, and 3) Section 4 derives a generic and flexible model extension based on the extracted elements. The paper focuses on the capabilities availability and reliability, because of their important role for most of resilience aspects, e.g., the enhanced capability dependability, especially in the area of upcoming exascale systems and the increasing number of components [9]. Related domains, like performance or energy efficiency, are under investigation at the moment to prepare them for application in capability effect analysis.

\section{Related Work}

This section investigates suitability of related work to model capability effect characteristics and their interplay to facilitate capability effect analysis. Related work is split in two groups, one focusing on resilience related aspects, and one on modeling HPC infrastructures in general. For each group, the general investigation is illustrated by representative examples.

The first group contains a lot of mature approaches that address certain detailed aspects of resilience very well. Nevertheless, all of them have at least one of the following drawbacks: 1) focusing on technical details and not on capability effects (wrong input), or 2) covering only (a few) resilience aspects and omitting other dimensions (not domain-spanning). Hence, neither the tool nor its underlying model can be used. An example for the first drawback is [10] that analyzes the impact of sub-optimal checkpointing (modification) on application efficiency (capability). An example for the second drawback is [11, developing a method to automatically select the best-fitting model to describe resource availability (capability), but without considering the interplay with other resilience capabilities or with capabilities of other dimensions.

The second group contains models to describe a network, computer system or a distributed system in general. The main drawback of this group is that it mainly models technical details and not capability effects and their interplay, especially not resilience capabilities. A representative example is the Common Information Model (CIM) [12. Even if some capabilities are described, e.g., the attribute NetworkAdapter.MaxSpeed, to our knowledge there are no resilience capabilities and most attributes describe technical aspects that are relevant only for modifications, e.g., the attribute MediaAccessDevice.MaxBlockSize. 


\section{Measurement and Modeling Methodology Overview}

This section forms a theoretical basis for the model extension development by considering the relevant measurement and methodologies in a condensed overview (Figure 2, based on 13/14). The commonalities of the considered elements, the model is required to cover in order to facilitate capability effect analysis, are extracted, as well. Commonality extraction focuses on the calculation results of the considered methodologies, because they express the intended effects (cf. Figure 1). To the contrary, details about modifications, e.g., the distinction of failures according to their causes, their severity, or their impact area [1314, are neglected, since they do not alter the measurement or calculation methodology, but only the used input, as further examples in this section illustrate.

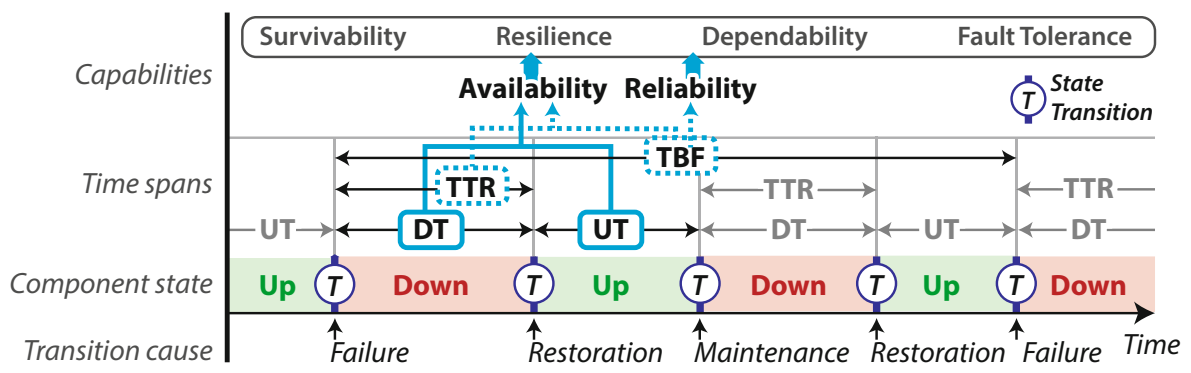

Fig. 2. Correlation of component states, state transitions, and quantification to derive availability and reliability figures

\subsection{Elementary Concepts}

A component is in the up state whenever it delivers a correct service or a system function as it is described by the functional specification [14]. A failure is a temporary or permanent termination of this ability and it causes a transition from correct to incorrect service or to down state [14. A restoration (also called replacement or repair) causes a transition from down to up state [15]16]. The time period a component is in the up state or down state is described by the Uptime $(\boldsymbol{U T})$ or Downtime $(\boldsymbol{D T})$, respectively. The time it takes to conduct a restoration is measured by the Time to Repair/Restore (TTR). The time span between the occurrence of two consecutive failures is described by the Time between Failures (TBF) [13. For all itemized measurements there are mean values labeled with a prefix $\boldsymbol{M}$, which are used to describe or calculate a component's availability and reliability among other capabilities. Especially the MTBF plays a central role, because of its strong correlation to failure interpretation, .e.g, it describes the frequency of (hardware) failures [15[17. An example of the aforementioned neglected details is the equality of $D T=T T R$ and $U T=T B F$ dependent on the consideration of scheduled maintenance [13 15], which does not influence the calculation methodology, but only the input values that are used to calculate availability and reliability. 


\subsection{Availability and Reliability}

Component availability describes the delivery of being in the up state, component reliability describes the continuousness of being in the up state under stated conditions over a specified period of time 2[14. There are many ways to structure and refine the two capabilities and their calculation rules, e.g., by distinguishing mission availability and work mission availability [18, or by describing different calculation rules for Total Availability, Scheduled System Availability, and Mean Time between Job Interrupts [19]. Since a modification's intended effects are expressed by calculation and measurement results (cf. Section 3) - e.g., reliability is calculated or expressed by $M T B F-$, we introduce a structuring that distinguishes capabilities and their calculation by the format of these calculation results. The structuring is depicted in Figure 3, showing the three result formats binary (yes/no), fraction $(\mathbb{R})$, and probability (\%), and the two influencing dimensions, which are described subsequently.

The first dimension is the line of sight ( $\mathrm{x}$-axis in Figure 3), describing whether the past or the future is analyzed: A retrospective view analyses existing data or empirical observations, which can be obtained in many ways, e.g., gathering field data or processing log files 20. The main challenge about a retrospective view is the underlying data, as it is of varying quality or very rare, since it is collected by observing parts failing while operating, a seldom event for well designed components [2]. In contrast, a prospective view creates predictions of future events by applying different probability distributions, like Weibull, Pareto or hyperexponential distributions. Depending on the objective, the existing empirical observation, and the considered component type, each distribution has specific advantages, e.g., Pareto is often advocated for lifetime estimation, whereby Weibull is more suitable for various resource availability data [2111].

The second dimension is the considered time (y-axis in Figure 3), describing whether only a certain point in time is analyzed or an interval between two points in time, whereby the interval can be finite or infinite. Since reliability describes the continuousness, it is always calculated for a period of time, whereby availability can also be calculated for a point in time, as depicted in Figure 3 by the thick lines at the top and at the right hand side of the possible formats.

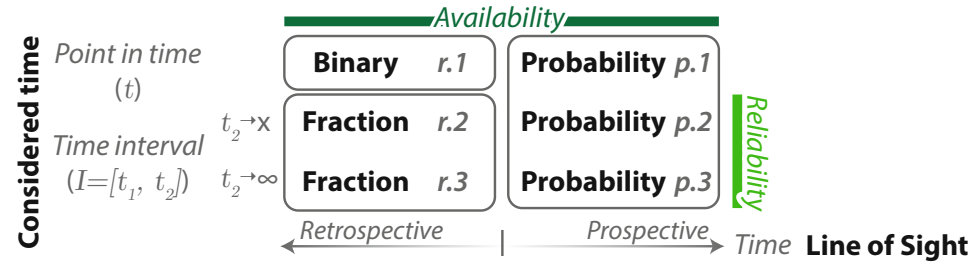

Fig. 3. Result types of availability and reliability calculation rules

Subsequently, a non-exhaustive list of examples is provided to further explain the differences, using the referencing labels (r.1)-(p.3) as depicted in Figure 3 ,

The binary result (r.1) states whether a component was available at a certain point in time in the past. An example for (r.3) is the steady-state availability 
$A_{s}$ describing the equilibrium behavior of a component. It is calculated by $A_{s}=$ $\frac{M T B F}{B F+M T T R}$ 22, using existing data for MTBF and MTTR. An example for (p.1) is the instantaneous availability or point availability $A(t)$, which is defined as the probability of finding a component in the up state at time $t[22$. It is calculated by an integral as described in [18 22. Another example for (p.1) is the "bathtub curve", describing the failure rate development over time dependent on the component's age describing parameter $t$ [15. An example for (p.2) is the interval availability, which describes the expected fraction of time the component is up in a given interval $I$ and is calculated by an integral. Reliability values in (p.2) and (p.3) are calculated by integrals and probability distributions as described by 2 .

In summary, every way to calculate availability or reliability generates a result having one of the three described formats.

\subsection{Composite Values}

As explained in Section 1 HPC infrastructures provide functionality by combining (several) components. Since the above investigated calculation rules mostly cover only one particular component, they have to be combined to results for combined components or sub systems, as well, leading to composite values. For instance, the composite availability value of a compute node is built up of the availability values of the contained components, like CPUs, internal bus, local memory, and network card. The composite availability value of the compute area in turn is build up of all compute node (composite) availability values.

Basically, composite values can be calculated by basic mathematical functions, like product, sum or mean. For instance, composite steady state availability can be calculated by multiplying the values of $\frac{f R_{i}}{f R_{i}+r R_{i}}$, whereby, $f R_{i}$ describes the failure rate, and $r R_{i}$ the repair rate of component $i$ [2]. It assumes that a combined component or sub system is available if and only if all of the contained components are available, or in other words, if one of the product operands is 0 (the component is not available), the overall result is also 0 . An example to calculate the composite reliability $R_{c}$ of a compound component is Part Count Analysis (PCA) that uses the sum operator as described by 23 .

\subsection{Redundancy Structures}

Compound nature of HPC infrastructures makes redundancy a strong tool to improve system reliability and availability: in the case a failure causes a transition to down state of a particular component, the system is able to continue to operate using a redundant component instead [9]. A system's redundancy structure can be described by boolean functions that use variables $c_{i}$ to describe that "component $c_{i}$ is available". For instance, the boolean function $\varphi=\left(c_{1} \vee c_{2}\right) \wedge c_{3}$ expresses that a system consisting of two redundant compute nodes $c_{1}, c_{2}$ and a network $c_{3}$ is available if the network and at least one of the two compute nodes is available [2]. A fault tree is a graphical representation of a redundancy structure, whereby the leaves represent availability (boolean variables), 
and inner nodes contain functions to calculate the overall system availability by processing the leave values up to the root.

\section{An Integrated Model}

This section extends the meta model described in 78 in two consecutive steps:

1) derive a set of criteria from the extracted commonalities (cf. Section 3 );

2) extend the model guided by the derived criteria set.

\subsection{Model Extension Criteria}

The development of the extended meta model was guided by a set of criteria. To apply the same development methodology as for the existing meta model, in this first step, a set of criteria is derived that guides the model extension. The criteria are labeled as C.R[1-6] to emphasize the commonality to [7] (expressed by $C$-riterion), but at the same time, to show the focus on resilience (expressed by $R$-esilience).

C.R1 - Different Calculation Rules and Result Types. There are several ways to calculate availability and reliability values, whose result is of one of the types binary, fraction, and probability (cf. Section 3.2). The neta model has to cover the result types and their manifold calculation rules on a component level. Additionally, it has to respect that every calculation rule has a different set of parameters,e.g., standard deviation, shape parameter, or a point in time.

C.R2 - Composite Values. Respecting the composite nature of HPC infrastructures requires the meta model to support the different ways of calculating composite values (cf. Section [3.3). To cover real world elements sufficiently accurate, calculation rules must be definable on a component level.

C.R3 - Redundancy Handling. Sufficient coverage of redundancy aspects requires the meta model to describe fault trees. To foster time and cost saving, analysis should be able to start with the most critical components [2] and hence, covering component criticality is required.

C.R4 - Decision-Making. To support (manual) decision-making and foster automatic problem solving, for every capability an improvement direction is necessary. For instance, an increase of $M T B F$ is an improvement, whereby a decrease of MTTR is an improvement.

C.R5 - Multiple Data Sources. To address the varying quality, uncertainty, incompleteness, and rareness of data used by retrospective and prospective analysis (cf. Section 3.2), the meta model has to support different data sources on component level. For instance, there are reliable vendor specifications for a CPU, but only incomplete empirical observations for the local memory.

C.R6 - Inherited Criteria. The extended meta model's criteria are $C 1-$ Real World Element Selection, C2-Multiple Levels of Model Granularity, C3Openness and Flexibility, and C4-Simplicity [7]. To ensure that the resulting meta model fulfills these origin criteria, the extension must fulfill them as well. 


\subsection{Extending the Meta Model by Resilience Aspects}

The meta model extension, whose result is depicted in Figure 4 as UML class diagram, is subsequently described, guided by the criteria C.R1-C.R6.

Fulfillment of C.R1 - The result types binary, fraction, and probability, are represented by interfaces in the value package. The ProbabilityValue is sub classed to emphasize different probability distributions like Weibull or Pareto, and to ensure by type safety that only results of the same distribution are processed together. Different parameter sets per calculation are handled by the key-value map parameter each interface method uses.

Fulfillment of C.R2 - The interfaces in the package compositeValue express the different ways to calculate composite values, whereby some simple operations are indicated. The interfaces are implemented by the Composite class that represents a (sub tree) root in the HPC infrastructure modeling tree [7/8].

Fulfillment of C.R3 - The Component class is extended by an attribute criticalityLevel, whose value can be set by applying existing approaches like fault injection 24] or CIM database analysis. To foster reusability of boolean functions, they can be prepared by the class RedundancyFunction and used by different Component objects.

Fulfillment of C.R4 - A Capability's improvement direction is described by an enumeration attribute improvementDirection, which is a sufficient data structure, since there can only be one improvement direction at the same time.

Fulfillment of C.R5 - To respect different data situations per component, every Component is associated with a AnalysisRuntimeCapabilityValue that implements one of the different capability value types. These values can be

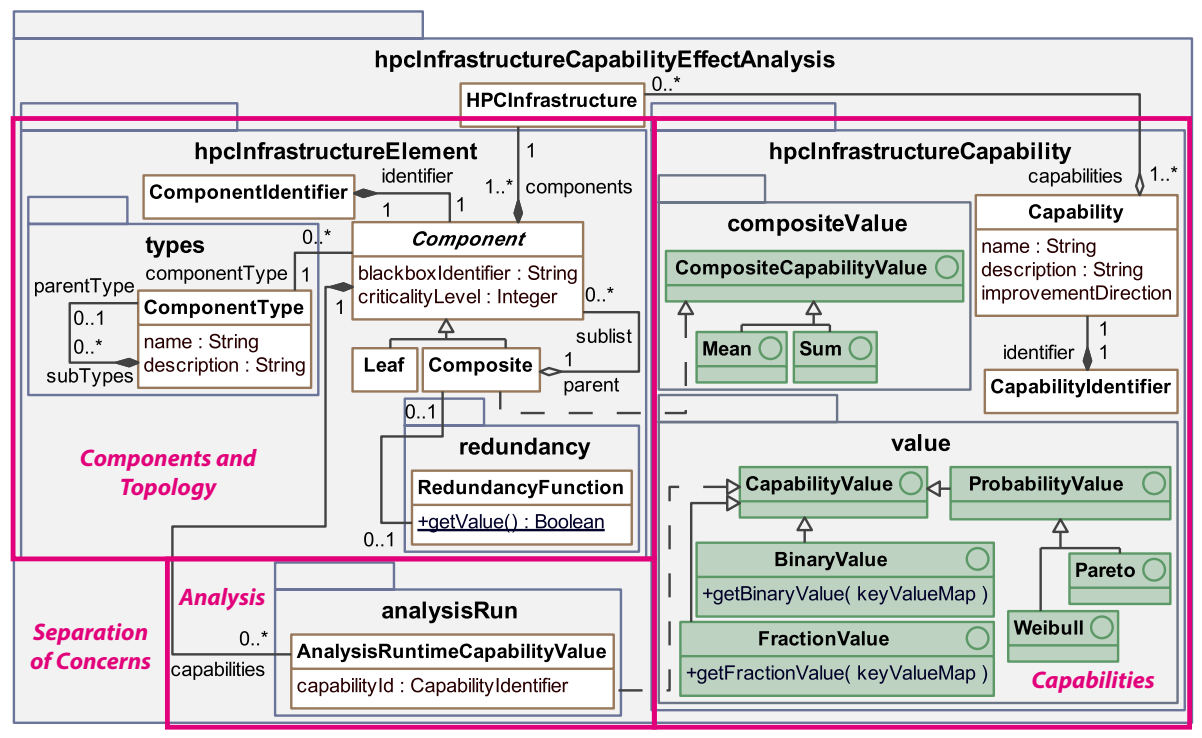

Fig. 4. UML class diagram of the capability model 
provided by different calculation rules, e.g., the availability of a storage Component can be "calculated" as fixed value provided by a vendor's specification, whereby the availability of a CPU Component can be calculated by a bathtub curve.

Fulfillment of C.R6 - The meta model extension fulfills the four origin criteria, because 1) it focuses on capability effects and hence, results in a valid model $(C 1), 2)$ it does not hamper the black box approach $(C 2), 3)$ it follows the Separation of Concerns (SoC) of the meta model, marked by the three rectangles in Figure 4, since the interfaces are only loosely coupled, and the set of prepared interfaces can flexibly enhanced by individual ones (C3), and 4) it supports the tree based modeling of an HPC infrastructure $(C 4)$.

\section{Conclusion and Future Work}

Capability effect analysis examines the domain-spanning cascading of a modification's intended effect within an entire HPC infrastructure to support the decision about the investigation and execution of a modification: only if the modification's intended effects produce an improvement as intended of the entire HPC infrastructure, the modification is executed. In [78] a meta model is presented, dedicated to support capability effect analysis, but lacking resilience consideration. This paper extends the meta model by resilience aspects in three steps: 1) give a condensed overview of relevant methodologies, 2) extracts their commonalities, and 3) derive a generic and flexible meta model extension based on the extracted elements. A Model Driven Development (MDD) process uses the developed meta model to generate a graphical editor that supports modeling (complex) HPC infrastructures and handling resilience aspects for capability effect analysis. The created HPC infrastructure model in turn serves as input for generating optimization code to address the optimization problem noted in Section 1. This process is currently applied to analyze the SuperMUC at the LRZ, a three PetaFlop/s HPC system.

\section{References}

1. Elliott, J., et al.: Combining Partial Redundancy and Checkpointing for HPC. In: Proc. of the 32th Intl. IEEE Conf. on Distributed Computing Systems, ICDCS (2012)

2. Eusgeld, I., Fechner, B., Salfner, F., Walter, M., Limbourg, P., Zhang, L.: Hardware Reliability. In: Eusgeld, I., Freiling, F.C., Reussner, R. (eds.) Dependability Metrics. LNCS, vol. 4909, pp. 59-103. Springer, Heidelberg (2008)

3. Chen, D., et al.: Looking Under the Hood of the IBM Blue Gene/Q Network. In: Proc. of the Intl. ACM/IEEE Conf. on High Performance Computing, Networking, Storage and Analysis, SC 2012 (2012)

4. Meswani, M., et al.: Modeling and Predicting Disk I/O Time of HPC Applications. In: Proc. of the High Performance Computing Modernization Program Users Group Conf., HPCMP-UGC (2010)

5. Broadbent, M., Weill, P.: Management by Maxim: How Business and IT Managers Can Create IT Infrastructures. Sloan Management Review 38(3), 77-92 (1997) 
6. Song, H., et al.: Availability Modeling and Evaluation on High Performance Cluster Computing Systems. Journal of Research and Practice in Information Technology $38(4), 317-335$ (2006)

7. Straube, C., Kranzlmüller, D.: A Meta Model for the Analysis of Modification Effects onto HPDC Infrastructures. Submitted to 27th Intl. Conf. on Architecture of Computing Systems, ARCS 2014 (2014)

8. Straube, C., Kranzlmüller, D.: An IT-Infrastructure Capability Model. In: Proc. of the 10th ACM Conf. on Computing Frontiers, CF 2013 (2013)

9. Jones, W., et al.: Application Monitoring and Checkpointing in HPC: Looking Towards Exascale Systems. In: Proc. of the 50th Annual Southeast Regional Conf. (2012)

10. Jones, W., et al.: Impact of Sub-Optimal Checkpoint Intervals on Application Efficiency in Computational Clusters. In: Proc. of the 19th Intl. ACM Symposium on High Performance Distributed Computing, HPDC 2010 (2010)

11. Nurmi, D., Brevik, J., Wolski, R.: Modeling Machine Availability in Enterprise and Wide-Area Distributed Computing Environments. In: Cunha, J.C., Medeiros, P.D. (eds.) Euro-Par 2005. LNCS, vol. 3648, pp. 432-441. Springer, Heidelberg (2005)

12. DMTF: Common Information Model (CIM) Metamodel (Specification). Technical Report DSP0004, Distributed Management Task Force, DMTF (2012)

13. Lu, C.: Failure Data Analysis of HPC Systems. CoRR abs/1302.4779 (2013)

14. Avizienis, A., et al.: Fundamental Concepts of Dependability. Technical report, University of California, Los Angeles, UCLA (2010)

15. Verbrugge, S., et al.: General Availability Model for Multilayer Transport Networks. In: Proc. of the 5th Intl. Workshop on Design of Reliable Communication Networks, DRCN 2005 (2005)

16. Pedicini, G., Green, J.: SPOTlight on Testing: Stability, Performance and Operational Testing of LANL HPC Clusters. In: Proc. of the Intl. ACM/IEEE Conf. for High Performance Computing, Networking, Storage and Analysis, SC 2011 (2011)

17. Boukerche, A., et al.: Towards Building a Highly-Available Cluster Based Model for High Performance Computing. In: Proc. of the 20th Intl. Parallel and Distributed Processing Symposium, IPDPS 2006 (2006)

18. Birolini, A.: Reliability Engineering: Theory and Practice. Springer (2007)

19. Stearley, J.: Defining and Measuring Supercomputer Reliability, Availability, and Serviceability (RAS). In: Proc. of the Linux Clusters Institute Conf. (2005)

20. Chandler, C., et al.: Towards Resilient High Performance Applications Through Real Time Reliability Metric Generation and Autonomous Failure Correction. In: Proc. of the Workshop on Resiliency in High Performance (2009)

21. Xu, J., et al.: Networked Windows NT system field failure data analysis. In: Proc. of the Intl. Pacific Rim Symposium on Dependable Computing (1999)

22. Trivedi, K.: Probability and Statistics with Reliability Queuing and Computer Science Applications. John Wiley \& Sons, Inc. (2001)

23. Military Handbook: Reliability Prediction of Electronic Equipment. Technical Report MIL-HDBK-217F, Department of Defense (1991)

24. Bagchi, S., et al.: Dependency Analysis in Distributed Systems using Fault Injection: Application to Problem Determination in an e-commerce Environment. In: Proc. of the 12th Intl. Workshop on Distributed Systems, DSOM 2001 (2001) 